\title{
Diagnosis and treatment of polycystic liver disease, presentation of a clinical case
}

\section{Abstract}

We present a clinical case of autosomal dominant polycystic kidney disease whit polycystic liver disease, in a 50-year-old asymptomatic woman. She was evaluated by urology, nephrology and hepatology, and an expectant management and periodic control was decided.

Keywords: polycystic liver disease, polycystic kidney disease, abdominal ultrasound, abdominal pain.
Volume 10 Issue 6 - 2019

\author{
Robin German Prieto Ortiz,' Jhon Edison \\ Prieto Ortiz ${ }^{2}$ \\ 'Department of Gastroenterology Service, Center for Hepatic \\ and Digestive Diseases, Colombia \\ ${ }^{2}$ Hepatology Service, Center for Hepatic and Digestive Diseases, \\ Colombia
}

\section{Correspondence: Robin German Prieto Ortiz, Department of Gastroenterology Service, Center for Hepatic and Digestive Diseases, Colombia, Email rgprietoo@hotmail.com}

Received: September 26, 2019 | Published: December 13, 2019
Abbreviations: PLD, polycystic liver disease; ADPKD, autosomal dominant polycystic kidney disease

\section{Introduction}

Polycystic Liver Disease (PLD), is an autosomal dominant disease, which in most cases is associated with autosomal dominant polycystic kidney disease (ADPKD), and less frequently occurs in isolation. They have a different genetic cause, but a similar pathological development. ADPKD has a prevalence of 0.13 to $0.52 \%$, with PLD being the most common extrarenal manifestation, with a prevalence of $58 \%$ in young people, $94 \%$ in adults. Isolated PLD has an incidence of less than $0.01 \%{ }^{1,2}$

\section{Case report}

50-year-old asymptomatic woman, with a history of hyperprolactinemia secondary to pituitary microadenoma, and infertility. In medical check-up they find hepatomegaly, so they request ultrasound, with finding of PLD. CT and abdominal resonance confirm the diagnosis (Figures 1-C). Digestive endoscopy shows extrinsic compression (Figure 1D). With a normal renal and hepatic profile, it is decided jointly with urology, nephrology and hepatology, expectant management and periodic control.
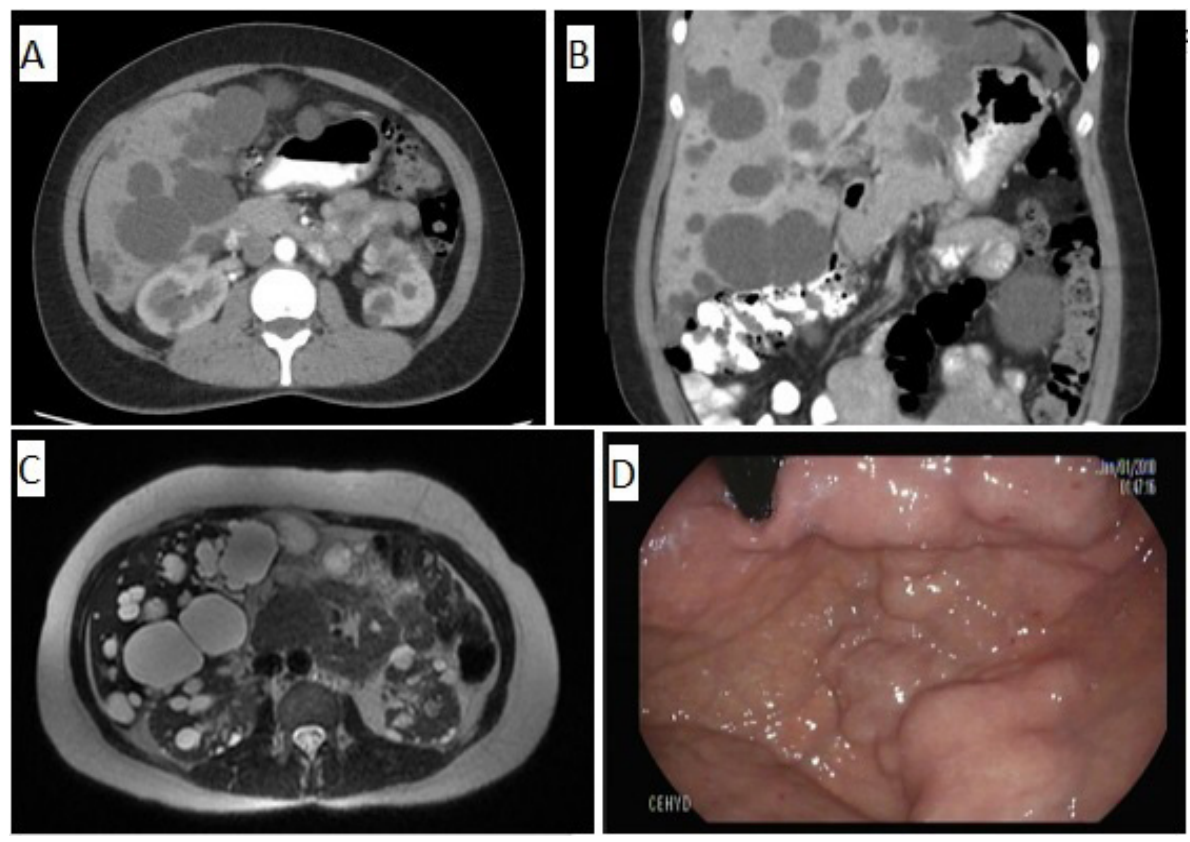

Figure I A-B Axial and coronal section, in which multiple hepatic cysts are observed. C. especially renal cysts are observed. D. In the retrovision of the upper digestive endoscopy, an image is observed that may suggest gastric varices, but which actually corresponds to the extrinsic compression caused by hepatic cysts. 


\section{Discussion}

Ravine criteria, allow to determine the presence of ADPKD according to the age of the patient and the number of renal cysts (Presence of at least 2 unilateral or bilateral cysts in patients under 30 years. Presence of two cysts in each kidney in patients between 30-50 years, or presence of at least 4 cysts in each kidney in patients over 60 years). In patients with isolated PLD, the presence of more than 20 cysts determines the diagnosis. Most cases are diagnosed incidentally, as in our patient, and may remain asymptomatic until the fourth decade of life. The main clinical manifestations are due to bloating and abdominal pain, infection and rupture of the cysts. ${ }^{3}$

There is no consensus regarding the management of this pathology, but clinical follow-up should always be performed. The therapy is aimed at decreasing the size of the cysts, through the use of somatostatin analogues. Aspiration and sclerotherapy, resection of the roof of the cyst, and liver resection, are the most commonly used interventional methods, and in extreme cases liver transplantation is necessary. 4,5

\section{Conclusion}

Knowing this about ADPKD and PLD, the physician must make oriented decisions to provide adequate care to these patients. An adequate diagnosis should be made that includes genetic studies. Adequate follow-up should be carried out to detect the presence of early complications, and according to them, perform the most appropriate treatment for each patient. The possibility of genetic counseling should be evaluated. And should always keep in mind that the patient should receive an interdisciplinary approach for their best care.

\section{Acknowledgments}

None.

\section{Conflict of interest}

The author declares no conflict of interest.

\section{References}

1. Ayala A, Aguilar JC, Jiménez F, Serpa F. Surgical treatment of the disease autosomal dominant polycystic liver number of cases. Rev Colomb Cir. 2015;30:139-145.

2. Montaña A, Patiño N, Larrate $\mathrm{C}$, et al. Update in polycystic kidney disease. Rev Fac Med. 2018;66(1):10716.

3. Barbado Cano A, Moreno López M, Olveira Martín A, et al. Ultrasoundguided percutaneous drainage and sclerotherapy in a patient with isolated autosomal dominant polycystic liver disease. Rev Esp Enferm Dig. 2015;107:175-177.

4. Khan S, Dennison A, Garcea G. Medical therapy for polycystic liver disease. Ann R Coll Surg Engl. 2016;98(1):18-23.

5. Chandok N. Polycystic liver disease: a clinical review. Annals of Hepatology. 2012;11(6):819-826. 